\title{
Estudo do nitreto de alumínio para aplicações termo-mecânicas
}

\section{(Study of aluminum nitride for thermomechanical applications)}

\author{
S. A. Baldacim ${ }^{1}$, O. M. M. Silva ${ }^{1}$, C. Santos ${ }^{2}$, C. R. M. Silva ${ }^{1}$ \\ ${ }^{I} C T A$ - IAE - Divisão de Materiais \\ ${ }^{2}$ Demar, Faenquil, Lorena, SP \\ Pça. Mar. do Ar Eduardo Gomes, 50, Vila das Acácias, S. J. dos Campos, SP 12228-904 \\ sandro.baldacim@ifi.cta.br
}

\begin{abstract}
Resumo
O nitreto de alumínio é muito utilizado como material semicondutor em dispositivos óptico-eletrônicos na qual sua alta condutividade térmica permite uma boa dissipação de calor. Por outro lado, devido à alta resistência mecânica e boa estabilidade estrutural à alta temperatura, tem sido crescente o seu estudo para aplicações termo-mecânica levando em considerações os avanços tecnológicos no processamento e caracterização destes materiais. Neste trabalho serão apresentados os resultados dos estudos de prensagem a quente do nitreto de alumínio aditivado com 5\%,10\% e 20\% de uma mistura contendo $\mathrm{Al}_{2} \mathrm{O}_{3}+\mathrm{Y}_{2} \mathrm{O}_{3}$. A massa específica foi determinada de acordo com a norma ASTM C20-87, as fases presentes por difração de raios X e as análises microestruturais por microscopia eletrônica de varredura. A microdureza e tenacidade à fratura foram avaliadas pelo método de indentação Vickers. Os resultados promissores alcançados de densificação, próximos à densidade teórica, e propriedades mecânicas, mostraram a viabilidade da utilização do AlN em aplicações estruturais.
\end{abstract}

Palavras-chave: nitreto de alumínio, propriedades físicas, propriedades mecânicas.

\begin{abstract}
Aluminum nitride is applied as semiconductor material in optical-electronic devices due to its high thermal conductivity and high heat dissipation. On the other hand, due to high mechanical strength and high-temperature structural stability this material has been studied for thermomechanical application. In this work the results of the physical and mechanical properties of aluminum nitride sintered by hot uniaxial pressing with 5\%,10\% and 20\% of $\mathrm{YAG}\left(\mathrm{Al}_{2} \mathrm{O}_{3}+\mathrm{Y}_{2} \mathrm{O}_{3}\right)$ additions are presented. The specific mass was determined by ASTM C20-87 standard, crystalline phases identified by X-ray diffraction and microstructural analysis performed by scanning electron microscopy. The microhardness and fracture toughness values were determined on the polished surface, at room temperature, using Vickers indentation method. The excellent results obtained for densification and mechanical properties show the viability of the use of aluminum nitride in structural applications.

Keywords: aluminum nitride, physical properties, mechanical properties.
\end{abstract}

\section{INTRODUÇÃO}

O nitreto de alumínio é um material cerâmico que não ocorre na natureza, mas pode ser sintetizado por reações em altas temperaturas, onde se destacam a deposição química em fase gasosa assistida por plasma reativo, redução carbotérmica e nitretação direta. É um material covalente de interesse significativo para o uso em diversas aplicações $[1,2]$.

O estudo no desenvolvimento do nitreto de alumínio tem sido crescente, principalmente por este ser um material muito aplicado na indústria da microeletrônica como dispositivo óptico eletrônico, devido à sua alta condutividade térmica e boa dissipação de calor. É um material semicondutor com ampla banda de energia e cristaliza-se na estrutura hexagonal wurtzita [3-6]. Entretanto, com o desenvolvimento tecnológico no processamento destes materiais, principalmente nas etapas de homogeneização e sinterização, o nitreto de alumínio passou a ser estudado e desenvolvido para aplicações termo-mecânica devido a alta resistência mecânica e excelente estabilidade a temperaturas elevadas [7-9].

Pode-se considerar, de uma maneira geral, que a utilização de pós finos das matérias-primas, associado com alta temperatura e alta pressão durante a sinterização, são considerações importantes a serem seguidas para a obtenção de matérias cerâmicos com alta densificação e boas propriedades mecânicas. Contudo, salientando a importância dos aditivos de sinterização, formadores da fase líquida, que é um fator essencial para a obtenção de materiais cerâmicos completamente densos [10].

Considerando-se os conceitos conhecidos sobre a sinterização via fase líquida, verifica-se que os aditivos são extremamente importantes para uma melhor densificação 
durante a sinterização e, conseqüentemente, para a obtenção de altos valores de propriedades mecânicas [11]. Durante os últimos anos, vários aditivos têm sido estudados, tais como $\mathrm{MgO}, \mathrm{Y}_{2} \mathrm{O}_{3}, \mathrm{Al}_{2} \mathrm{O}_{3}$, óxidos de terras raras, na tentativa de otimizar o processo e adequar as características microestruturais às propriedades desejadas $[12,13]$.

No caso do nitreto de alumínio, pode-se obter elevados resultados de massa específica relativa, densificando-o uniformemente, utilizando aditivos de sinterização baseado em combinações de óxido de terras raras ou $\mathrm{Y}_{2} \mathrm{O}_{3} \operatorname{com~} \mathrm{Al}_{2} \mathrm{O}_{3}$, na qual fornece um líquido eutético com menor temperatura de fusão [13, 14]. Assim sendo, com a utilização do $\mathrm{Y}_{2} \mathrm{O}_{3}$ com $\mathrm{Al}_{2} \mathrm{O}_{3}$ como aditivo de sinterização do nitreto de alumínio, espera-se uma alta densificação devido à formação de fase líquida a temperaturas mais baixas e a formação de fase intergranular YAG $\left(\mathrm{Y}_{3} \mathrm{Al}_{5} \mathrm{O}_{12}\right)$, que irá envolver os grãos de nitreto de alumínio de maneira uniforme, contribuindo para uma alta massa específica relativa.

Neste trabalho serão apresentados e discutidos os resultados dos estudos de prensagem a quente do nitreto de alumínio aditivado com YAG $\left(\mathrm{Al}_{2} \mathrm{O}_{3}+\mathrm{Y}_{2} \mathrm{O}_{3}\right)$, previamente misturado, visando aplicações termo-mecânica.

\section{MATERIAIS E MÉTODOS}

$\mathrm{Na}$ Tabela I estão mostradas as composições estudadas. Utilizou-se como base nitreto de alumínio (H. C. Starck) aditivado com uma mistura de óxido de ítrio (H. C. Starck) e alumina (Baikalox), na proporção de 5, 10 e $20 \%$.

Após estipuladas as composições, por meio da regra da mistura, iniciou-se a pesagem dos pós precursores em balança analítica. Em seguida realizou-se a etapa de moagem/homogeneização via úmido, em moinho de bolas, por um período de $24 \mathrm{~h}$, utilizando-se etanol absoluto.

Após esta etapa, as composições foram compactadas em matriz de aço, com camisa flutuante, com carga de $50 \mathrm{MPa}$, sendo em seguida submetidas a prensagem uniaxial a frio a $300 \mathrm{MPa}$. Esta etapa visa a obtenção de corpos-de-prova com maiores e mais uniformes valores de densidade a verde, permitindo uma boa resistência ao seu manuseio.

$\mathrm{O}$ processo de sinterização por prensagem uniaxial a quente $(\mathrm{HP})$ foi realizado sob atmosfera de nitrogênio, à temperatura de $1800^{\circ} \mathrm{C}$, com patamar de 30 minutos. Adotou-

Tabela I - Composições utilizadas neste trabalho. [Table I - Compositions utilized in this work.]

\begin{tabular}{ccc}
\hline Composições & $\begin{array}{c}\mathrm{AlN} \\
(\% \text { em peso })\end{array}$ & $\begin{array}{l}\mathrm{Y}_{2} \mathrm{O}_{3}+\mathrm{Al}_{2} \mathrm{O}_{3} \\
(\% \text { em peso })\end{array}$ \\
\hline A (AlN95YAG5) & 95 & 5 \\
B (AlN90YAG10) & 90 & 10 \\
C (AlN80YAG20) & 80 & 20 \\
\hline
\end{tabular}

se uma taxa de aquecimento de $20{ }^{\circ} \mathrm{C} / \mathrm{min}$. Completado o patamar, o forno foi desligado com o corpo-de-prova permanecendo no seu interior até a temperatura ambiente. Este ciclo, para o processamento destes materiais, foi utilizado por ter sido otimizado em trabalhos realizados anteriormente $[15,16]$.

Com relação às propriedades físicas, a massa específica foi determinada utilizando-se a norma ASTM C20-87, tomando-se em média três medidas de cada corpo-de-prova, e as fases presentes foram identificadas por difração de raios $\mathrm{X}$. As análises microestruturais das seções transversais dos corpos-de-prova sinterizados, polidas e atacadas com um reagente adequado, foram realizadas por microscopia eletrônica de varredura (MEV).

Com relação às propriedades mecânicas, determinouse a microdureza e a tenacidade à fratura, utilizando o método de impressão por indentação Vickers, no qual os corpos-de-prova foram submetidos a cinco indentações, com carga de $5,0 \mathrm{~kg}$, utilizando penetrador de diamante.

\section{RESULTADOS E DISCUSSÃO}

\section{Caracterização das matérias-primas}

As características dos pós de nitreto de alumínio (AlN), alumina $\left(\mathrm{Al}_{2} \mathrm{O}_{3}\right)$ e óxido de ítrio $\left(\mathrm{Y}_{2} \mathrm{O}_{3}\right)$ estão apresentadas na Tabela II. Estes dados foram fornecidos pelos respectivos fabricantes.

Os resultados obtidos pelas análises granulométricas realizadas nas matérias-primas como recebidas, utilizando o método de sedimentação gravimétrica, apresentaram distribuição estreita de tamanho de partículas, com tamanho médio de partículas em torno de 1,21 $\mu \mathrm{m}$ (AlN), $0,59 \mu \mathrm{m}\left(\mathrm{Y}_{2} \mathrm{O}_{3}\right)$ e $0,98 \mu \mathrm{m}\left(\mathrm{Al}_{2} \mathrm{O}_{3}\right)$.

Após o processo de mistura/homogeneização, as composições apresentaram uma distribuição de tamanho médio de partículas de forma estreita, com variação entre 0,12 a $1,40 \mu \mathrm{m}$. A característica de forma estreita é resistente ao crescimento excessivo de partículas e a formação de uma microestrutura grosseira, contribuindo para uma melhor densificação [15]. O tamanho médio de partículas foi de $0,52 \mu \mathrm{m}, 0,61 \mu \mathrm{m}$ e $0,66 \mu \mathrm{m}$ para as composições A (AlN95YAG5), B (AlN90YAG10) e C (AlN80YAG20), respectivamente. Este decréscimo no tamanho médio de partículas, quando comparado aos materiais de partida, pode promover uma maior densificação dos corpos-de-prova na etapa de sinterização.

Segundo Kingery [17], a força motriz para a densificação é favorecida pelo excesso de energia superficial que os pós exibem. Assim, quanto menor for a partícula, maior será a energia superficial e, conseqüentemente, maior será a força motriz para a densificação [17]. 
Tabela II - Características das matérias-primas utilizadas neste trabalho.

[Table II - Characteristics of raw material utilized in this work.]

\begin{tabular}{|c|c|c|c|c|c|c|}
\hline $\begin{array}{l}\text { Matéria- } \\
\text { prima }\end{array}$ & $\begin{array}{c}\text { Área } \\
\text { específica } \\
\left(\mathrm{m}^{2} / \mathrm{g}\right)\end{array}$ & Fabricante & Grade & Lote & $\begin{array}{c}\text { Elementos } \\
\text { presentes } \\
(\% \text { em peso })\end{array}$ & $\begin{array}{c}\text { Massa } \\
\text { específica } \\
\left(\mathrm{g} / \mathrm{cm}^{3}\right)\end{array}$ \\
\hline AlN & 2,60 & H. C. Starck & B & $63121 / 96$ & $\begin{array}{c}\text { AlN }-98 \% \\
\text { O - } 1,1 \\
\text { Fe - } 800 \text { ppm } \\
\text { C - } 30 \text { ppm }\end{array}$ & 3,26 \\
\hline $\mathrm{Y}_{2} \mathrm{O}_{3}$ & 12,76 & H. C. Starck & $\begin{array}{c}\mathrm{C} \\
\text { (fino) }\end{array}$ & $2010 / 96$ & $\begin{array}{c}\mathrm{Y}_{2} \mathrm{O}_{3}-99,98 \\
\mathrm{Al}<2 \mathrm{ppm} \\
\mathrm{Fe}<1 \mathrm{ppm} \\
\mathrm{Ca}<0,5 \mathrm{ppm}\end{array}$ & 5,01 \\
\hline $\mathrm{Al}_{2} \mathrm{O}_{3}$ & 6,0 & Baikalox & CR-6 & - & $\begin{array}{c}\mathrm{Al}_{2} \mathrm{O}_{3}-99,9 \\
\mathrm{Na}^{2} 12 \mathrm{ppm} \\
\mathrm{K}<41 \mathrm{ppm} \\
\mathrm{Fe}<7 \mathrm{ppm} \\
\mathrm{Ca}<47 \mathrm{ppm}\end{array}$ & 3,20 \\
\hline
\end{tabular}

\section{Massa especifica}

A massa específica aparente foi determinada utilizando a norma ASTM C20-87, tomando-se em média três medidas. Determinou-se, também, a massa específica relativa obtida pela relação entre a massa específica aparente e a calculada pela regra das misturas. Os resultados estão apresentados na Tabela III.

Tabela III - Resultados obtidos de massa específica aparente e relativa.

[Table III - Results of specific mass apparent and relative.]

\begin{tabular}{ccc}
\hline Composição & $\begin{array}{c}\rho_{\text {aparente }} \\
\left(\mathrm{g} / \mathrm{cm}^{3}\right)\end{array}$ & $\begin{array}{c}\rho_{\text {relativa }} \\
(\%)\end{array}$ \\
\hline A (AlN95YAG5) & 3,19 & 96,80 \\
B (AlN90YAG10) & 3,24 & 97,70 \\
C (AlN80YAG20) & 3,36 & 98,90 \\
\hline
\end{tabular}

Analisando os resultados obtidos, observou-se que o aumento na porcentagem de aditivos promoveu um acréscimo nos valores de massa específica aparente para todas as composições. Isto se deve a maior quantidade de fase líquida formada, que intensifica o mecanismo de rearranjo de partículas e o processo de soluçãoreprecipitação [11].

Durante o processo de sinterização por prensagem uniaxial a quente, a aplicação de pressão externa, associada à alta temperatura, acelera a cinética de densificação pelo aumento do contato entre as partículas e pelo maior empacotamento destas. Segundo Petzow [18], o acréscimo da taxa de densificação, com a utilização de pressão externa, pode estar relacionada ao aumento de solubilidade nos pontos de contato, auxiliando nas etapas de rearranjo e solução-reprecipitação dos grãos.

\section{Difração de raios $X$}

As fases cristalinas, identificadas por difração de raios $\mathrm{X}$, foram basicamente referentes ao nitreto de alumínio ( $\beta$ AIN) e a formação de fases intergranulares $\mathrm{Y}_{3} \mathrm{Al}_{5} \mathrm{O}_{12}(\mathrm{YAG})$ e $\mathrm{Al}_{3} \mathrm{O}_{3} \mathrm{~N}$ (oxinitreto de alumínio), correspondente aos aditivos de sinterização utilizados.

As fases intergranulares estão relacionadas, provavelmente, à taxa de resfriamento que possibilitou uma maior nucleação e cristalização destas fases em meio a fase líquida. Sabe-se que a fase líquida formada durante a sinterização solidifica-se, no resfriamento, na forma amorfa ou parcialmente cristalina [18].

A não constatação, ao nível de resolução do equipamento utilizado, de outros óxidos que compõem as composições pode estar associada às quantidades envolvidas que, provavelmente, deve ter entrado em solução sólida ou solidificado na forma amorfa.

\section{Análise microestrutural}

Para análise microestrutural por microscopia eletrônica de varredura, os corpos-de-prova foram polidos e atacados termicamente com um reagente adequado $(\mathrm{NaOH}$ e $\mathrm{KOH}$, 1:1, a $500^{\circ} \mathrm{C}, 5 \mathrm{~min}$ ). Não se utilizou análise por EDS nesta etapa em função de problemas operacionais.

O nitreto de alumínio apresenta em sua superfície oxigênio que reage com os aditivos para formar a fase líquida importante para densificação. Com a utilização de uma mistura $\mathrm{Y}_{2} \mathrm{O}_{3}+\mathrm{Al}_{2} \mathrm{O}_{3}$ como aditivos de sinterização, as fases intergranulares YAG (garnets) e $\mathrm{Al}_{3} \mathrm{O}_{3} \mathrm{~N}$ foram formadas, como identificadas nas difrações de raios $\mathrm{X}$. Estas fases cristalizam-se nos contornos de grãos e junções triplas de grãos do nitreto de alumínio, caracterizadas por uma coloração mais escura, conforme se pode verificar nas análises microestruturais mostradas pelas Figs. 1-2. 


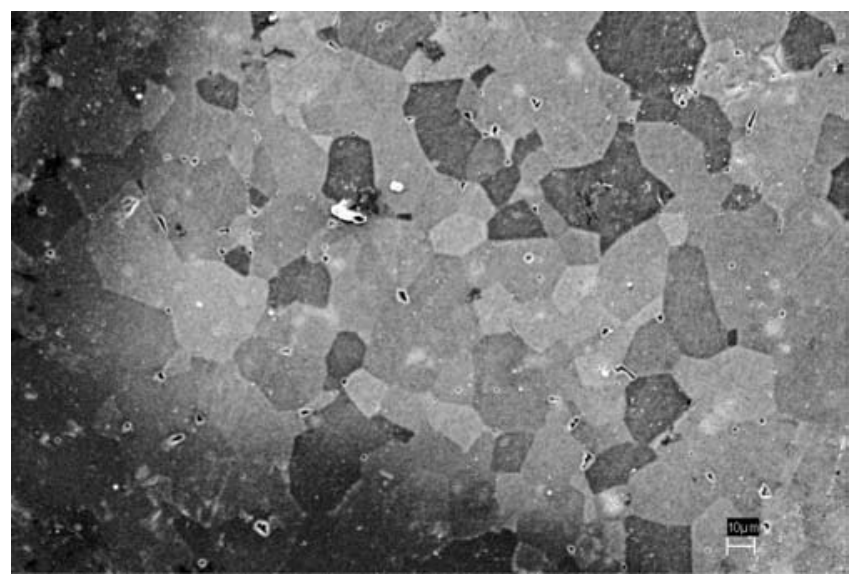

Figura 1: Fotomicrografia obtida por MEV da superfície do nitreto de alumínio com $10 \%$ de $\mathrm{Y}_{2} \mathrm{O}_{3}+\mathrm{Al}_{2} \mathrm{O}_{3}$. Observa-se a presença de grãos de AlN (regiões claras) caracterizado por estrutura hexagonal e grãos de fases intergranulares (regiões mais escuras) caracterizado por plaquetas.

[Figure 1: SEM photomicrographs of surface of aluminum nitride hot pressed sintered with $10 \mathrm{wt} \% \mathrm{Y}_{2} \mathrm{O}_{3}+\mathrm{Al}_{2} \mathrm{O}_{3}$ sintering aids. Observed the presence of AlN grains (white regions) characterized by hexagonal structure and intergranular phases (dark regions) grains characterized by platelets.]

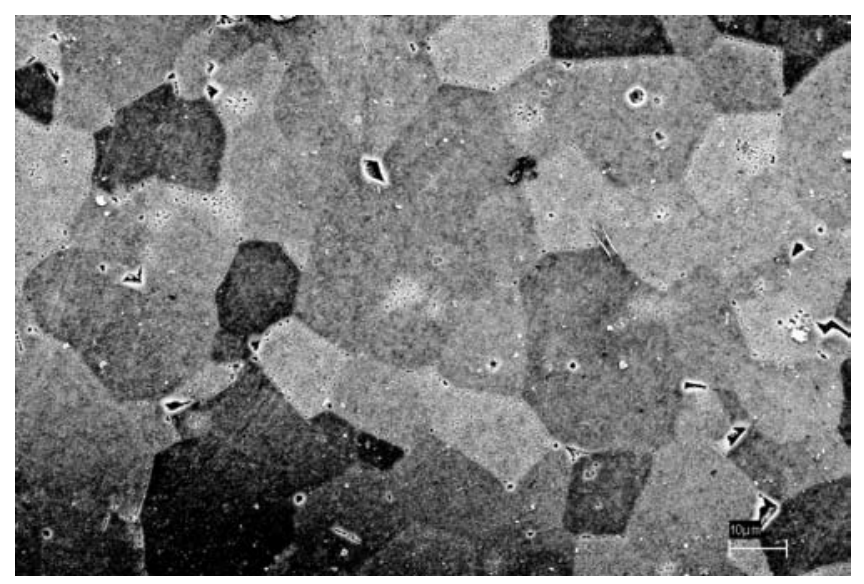

Figura 2: Fotomicrografia obtida por MEV da superfície do nitreto de alumínio com $10 \%$ de $\mathrm{Y}_{2} \mathrm{O}_{3}+\mathrm{Al}_{2} \mathrm{O}_{3}$. Observa-se a presença de grãos de AlN (regiões claras) caracterizado por estrutura hexagonal e grãos de fases intergranulares (regiões mais escuras) caracterizado por plaquetas.

[Figure 2: SEM photomicrographs of surface of aluminum nitride hot pressed sintered with $10 \mathrm{wt} \% \mathrm{Y}_{2} \mathrm{O}_{3}+\mathrm{Al}_{2} \mathrm{O}_{3}$ sintering aids. Observed the presence of AlN grains (white regions) characterized by hexagonal structure and intergranular phases (dark regions) grains characterized by platelets.]

Os aditivos óxidos removem o oxigênio da superfície do nitreto de alumínio formando as fases intergranulares. Com o aumento dos aditivos, as fases intergranulares podem se apresentar na forma de plaquetas tão grandes que podem até inibir o contato entre os grãos de nitreto de alumínio [5]. Estas plaquetas podem ser do tipo YAG e/ou 7 AlN. $\mathrm{Al}_{2} \mathrm{O}_{3}$, cristalizados na fase líquida e circundados por cristais de nitreto de alumínio esféricos [5]. Na Fig. 3, observa-se uma superfície de fratura com pequenas regiões de plaquetas de fases intergranulares.

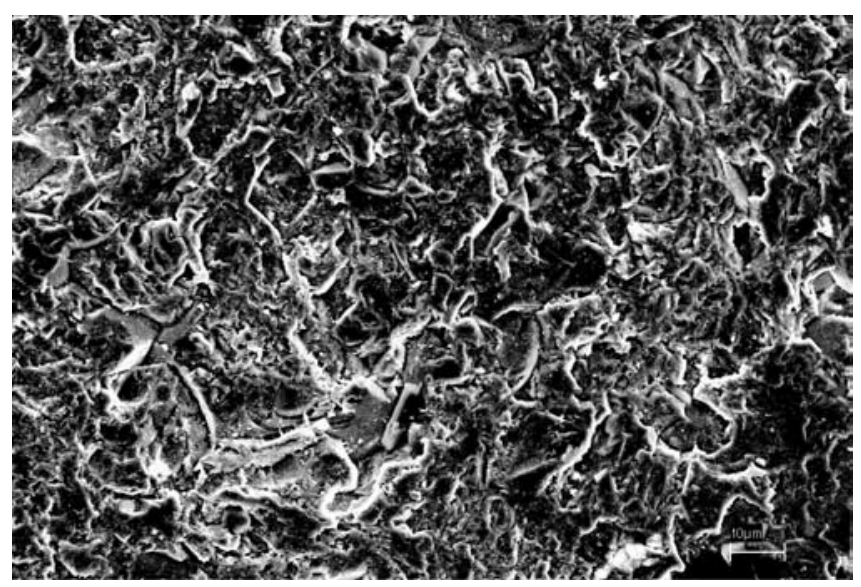

Figura 3: Fotomicrografia obtida por $\mathrm{MEV}$ da superfície do nitreto de alumínio com $20 \%$ de $\mathrm{Y}_{2} \mathrm{O}_{3}+\mathrm{Al}_{2} \mathrm{O}_{3}$. Observa-se uma superfície de fratura com pequenas regiões de plaquetas de fases intergranulares.

[Figure 3: SEM photomicrographs of surface of aluminum nitride hot pressed sintered with $20 \mathrm{wt} \% \mathrm{Y}_{2} \mathrm{O}_{3}+\mathrm{Al}_{2} \mathrm{O}_{3}$ sintering aids. Observed the fracture surface with small platelets regions of intergranular phases.]

\section{Microdureza Vickers e tenacidade à fratura}

A Tabela IV apresenta os resultados de microdureza Vickers e tenacidade à fratura, obtidos pelo método de indentação. Para microdureza, os corpos-de-prova foram submetidos a cinco impressões, com carregamento de 5,0 $\mathrm{kg}$, sendo a primeira na superfície e as demais nas seções transversais, caracterizando um perfil de dureza da superfície para o núcleo. Para a tenacidade à fratura, tomou-se a média de cinco impressões, determinando o valor médio do comprimento das duas trincas que se originaram do vértice da diagonal de impressão Vickers.

Analisando os resultados obtidos em função do desviopadrão, observam-se valores constantes de microdureza

Tabela IV - Resultados de microdureza Vickers e tenacidade à fratura.

[Table IV - Results of hardness Vickers and fracture toughness.]

\begin{tabular}{ccc}
\hline Composição & $\begin{array}{c}\text { MicrodurezaVickers } \\
(\mathrm{GPa})\end{array}$ & $\begin{array}{c}\mathrm{K}_{\mathrm{lc}} \\
\left(\mathrm{MPa} \mathrm{m}^{1 / 2}\right)\end{array}$ \\
\hline A (AlN95YAG5) & $15,29 \pm 0,36$ & $4,92 \pm 0,15$ \\
B (AlN90YAG10) & $16,31 \pm 0,39$ & $5,37 \pm 0,14$ \\
C (AlN80YAG20) & $17,16 \pm 0,28$ & $5,76 \pm 0,23$ \\
\hline
\end{tabular}


Vickers, da superfície para o núcleo nos corpos-de-prova, indicando uma boa homogeneidade do material.

Observa-se que os valores de microdureza Vickers aumentam com o acréscimo do nível de densificação, avaliado pela massa específica aparente, estando diretamente ligado ao aumento da quantidade de aditivos utilizados.

Estudos [15] também demonstraram que o aumento de fase intergranular pode ocasionar um acréscimo na microdureza, devido ao aumento da massa específica aparente. Entretanto, esta relação é válida desde que não se forme uma grande quantidade de fase vítrea ou que haja um crescimento exagerado de grãos, $o$ que não ocorreu neste trabalho.

Os altos valores de tenacidade à fratura estão levemente superiores aos encontrados na literatura (3 - 4,5 MPa.m $\left.{ }^{1 / 2}\right)[7$, 8]. Estes valores podem ser influenciados pela morfologia de grãos, a qual está diretamente ligada a quantidades de aditivos. Grãos ou plaquetas com aspectos alongados, observados por microscopia eletrônica de varredura, podem proporcionar a atuação do mecanismo de tenacificação conhecido como deflexão de trinca [19], contribuindo de maneira significativa para o aumento da tenacidade à fratura.

Outro fator para o aumento da tenacidade à fratura pode estar relacionado com a cristalização da fase intergranular, na qual aumenta o nível de tensão residual da microestrutura, devido aos diferentes coeficientes de expansão térmica, provocando microtrincas que servem de obstáculos para a propagação de trincas, atuando como reforço [19].

\section{CONCLUSÕES}

Os resultados obtidos de massa específica, fases presentes, aspectos microestruturais e propriedades mecânicas mostraram ser dependentes da quantidade de aditivos e do processo de sinterização por prensagem a quente utilizada.

Com o aumento na quantidade de aditivo de sinterização observou-se um acréscimo na taxa de densificação, devido a maior quantidade de fase líquida formada e, conseqüentemente, um acréscimo nos valores de microdureza.

As fases cristalinas do nitreto de alumínio $\mathrm{e}$ intergranulares $\mathrm{Y}_{3} \mathrm{Al}_{5} \mathrm{O}_{12}$ (YAG) e $\mathrm{Al}_{3} \mathrm{O}_{3} \mathrm{~N}$ (oxinitreto de alumínio) foram identificadas por difração de raios X. Apesar de não ser possível realizar análises por EDS, observou-se por microscopia eletrônica de varredura grãos de nitreto de alumínio (regiões claras) e fases intergranulares que cristalizaram nos contornos de grãos e junções triplas de grãos do nitreto de alumínio (regiões escuras).

Os altos valores de tenacidade à fratura podem estar relacionados à morfologia dos grãos (plaquetas) e a presença de fase intergranular cristalizada, ativando mecanismos de tenacificação como deflexão de trincas e microtrincamento. Ambos mecanismos servem de obstáculo para as trincas, consumindo uma energia maior para a sua propagação e, conseqüentemente, atuando com reforço.

Os excelentes resultados alcançados de densificação, próximos à densidade teórica, e propriedades mecânicas, mostraram a eficiência e viabilidade do uso de nitreto de alumínio utilizando um mistura $\mathrm{Y}_{2} \mathrm{O}_{3}+\mathrm{Al}_{2} \mathrm{O}_{3}$ como aditivo de sinterização, podendo obter materiais cerâmicos para ser utilizado em aplicações termo-mecânica.

Entretanto, etapas suplementares de caracterização estão em andamento para uma análise mais completa e criteriosa como, por exemplo, ensaios de fluência para verificar o comportamento destes materiais em temperaturas elevadas.

\section{AGRADECIMENTOS}

FAPESP - Fundação de Amparo à Pesquisa do Estado de S. Paulo, CTA/IAE/AMR - Divisão de Materiais, DEMAR/ FAENQUIL - Departamento de Materiais Cerâmicos.

\section{REFERÊNCIAS}

[1] F. Malengreau, M. Vermeersh, J. Mater. Res. 12, 11 (1997) 175.

[2] G. P. Johnston, D. M. Smith, J. Am. Ceram. Soc. 75, 12 (1992) 3465.

[3] Z. Q .Yang, L. L. He, Mater. Sci. Eng. A 323 (2002) 754.

[4] Y. Liu, Y. Wu, H. Zhou, Mater. Lett. 35 (1998) 232.

[5] C. F. Chen, M. E. Perisse, A. F. Ramires, J. Mater. Sci. 29 (1994) 1595.

[6] T. B. Jackson, A. V. Virkar, K. L. More, R. B. Dinwiddie, R. A. Cutler, J. Am. Ceram. Soc. 80, 6 (1997) 1421.

[7] H. Tamai, T. Hamauzu, A. Ryumon, K. Aoki, K. Oda, T. Yoshio, J. Am. Ceram. Soc. 83, 12 (2000) 3216.

[8] Y-H. Koh, J-J. Choi, H-E. Kim, J. Am. Ceram. Soc. 83, 2 (2000) 306.

[9] M. L. Panchula, J. Y. Ying, J. Am. Ceram. Soc. 86, 7 (2003) 1114.

[10] D. W. Richerson, Modern Ceramic Engineering, $2^{\text {nd }}$ Ed., New York (1992) p.569.

[11] R. M. German, Liquid Phase Sintering, Plenum Press, New York (1985).

[12] F. Boey, X. L. Song, Z. Gu, J. Mater. Proc. Technol. 89-90 (1999) 478.

[13] F. Boey, L. Cao, K. A. Khor, Acta Mater. 49 (2001) 3117.

[14] T. Shurakami, H. Nakano, J. Am. Ceram. Soc. 84, 3 (2001).

[15] S. A. Baldacim, Tese de Doutorado, Instituto de Pesquisas Energéticas e Nucleares - USP (2000).

[16] O. M. M. Silva, Diss. Mestrado, Instituto Tecnológico de Aeronáutica (ITA) (1996).

[17] W. D. Kingery, H. K. Bowen, D.R. Uhlmann, Introduction to ceramics, John Wiley \& Sons, New York (1976).

[18] W. A. Kaysser, G. Petzow, Recent Conception on Liquid Phase Sintering, Proc. Sintering Theory and Practice Conf. London (1984).

[19] R. Warren, Ceramic-matrix composites, Chapman and Hall, New York (1992).

(Rec. 01/07/2004, Rev. 03/06/2005, Ac. 04/11/2005) 